PROCEEDINGS OF THE AMERICAN MATHEMATICAL SOCIETY

Volume 124, Number 11, November 1996

\title{
SELF-SIMILARITY IN INVERSE LIMIT SPACES OF THE TENT FAMILY
}

\author{
MARCY BARGE, KAREN BRUCKS, AND BEVERLY DIAMOND
}

(Communicated by Mary Rees)

\begin{abstract}
Taking inverse limits of the one-parameter family of tent maps of the interval generates a one-parameter family of inverse limit spaces. We prove that, for a dense set of parameters, these spaces are locally, at most points, the product of a Cantor set and an arc. On the other hand, we show that there is a dense $G_{\delta}$ set of parameters for which the corresponding space has the property that each neighborhood in the space contains homeomorphic copies of every inverse limit of a tent map.
\end{abstract}

In 1967, R. F. Williams ([10]) proved that hyperbolic one-dimensional attractors are inverse limits of maps on branched one-manifolds. These attractors have the solenoid-like property of being everywhere locally homeomorphic with the product of a Cantor set and an arc. Also, for dissipation parameter near zero, most of the full attracting sets for maps in the Hénon family are homeomorphic with inverse limits of unimodal maps of the interval ([1]). Except at finitely many points (the points of a stable periodic orbit), these sets are locally homeomorphic with the product of a Cantor set and an arc (see the comment following Theorem 1).

Computer-generated pictures, at first glance, suggest that other one-dimensional (but non-hyperbolic) attractors might have a similar local structure. In particular, the transitive Hénon attractors appear to be, at most points, locally the product of a Cantor set and an arc. However, 'blowing up' computer pictures of these attractors usually indicates the presence of 'hooks' in the midst of regions that, under less scrutiny, look like a Cantor set of nearly parallel arcs.

In this paper we consider the local topological properties of a one-parameter family of conceptual models for the Hénon attractors, inverse limits of tent maps. We find the following: for a dense set of parameters, the inverse limit space is, except at finitely many points, the product of a Cantor set and an arc (Theorem 1 ). However, for a dense $G_{\delta}$ set of parameters, the inverse limit space is nowhere locally homeomorphic with the product of a Cantor set and an arc. In this second case, the inverse limit spaces display a remarkable form of self-similarity and local recapitulation of the entire family: not only does every open set in each space contain a homeomorphic copy of the entire space, each open set also contains a homeomorphic copy of every other inverse limit space appearing in the tent family (Corollary 6). In a forthcoming paper, we prove that the set of parameters for which this holds has full measure.

Received by the editors May 16, 1995.

1991 Mathematics Subject Classification. Primary 54F15, 58F03, 58F12.

The first author was supported in part by NSF-DMS-9404145. 
We introduce terminology, notation, and preliminary results as needed for the main results.

Suppose that $\left\{X_{i}\right\}_{i=1}^{\infty}$ is a collection of compact metric spaces and that for each $i, f_{i}: X_{i+1} \rightarrow X_{i}$ is a continuous map. The inverse limit space of $\left\{X_{i}, f_{i}\right\}_{i=1}^{\infty}$ (or simply, of $\left.\left\{f_{i}\right\}_{i=1}^{\infty}\right)$ is

$$
\left\{\underline{x}=\left(x_{0}, x_{1}, \ldots\right) \mid \underline{x} \in \prod_{i=1}^{\infty} X_{i}, f_{i}\left(x_{i+1}\right)=x_{i}, i \in \mathbb{N}\right\}
$$

and has metric $\underline{d}$ given by

$$
\underline{d}(\underline{x}, \underline{y})=\sum_{i=0}^{\infty} \frac{d_{i}\left(x_{i}, y_{i}\right)}{2^{i}}
$$

where for each $i, d_{i}$ is a metric for $X_{i}$ bounded by 1 . For each $i, \Pi_{i}$ will denote the restriction of the usual projection map from $\prod_{i=1}^{\infty} X_{i}$ into $X_{i}$ to the inverse limit space defined above. If $X_{i}=X$ and $f_{i}=f$ for all $i$, the inverse limit space is denoted by $(X, f)$, and the map $\hat{f}:(X, f) \rightarrow(X, f)$ defined by $\hat{f}\left(\left(x_{0}, x_{1}, \ldots\right)\right)=$ $\left(f\left(x_{0}\right), x_{0}, x_{1}, \ldots\right)$ is called the induced homeomorphism.

In the following, $I$ will denote the unit interval $[0,1]$. For $\lambda \in[1,2]$, define the following families of maps: the family of tent maps $T_{\lambda}: I \rightarrow I$ is defined by

$$
T_{\lambda}(x)= \begin{cases}\lambda x, & 0 \leq x \leq 0.5, \\ \lambda(1-x), & 0.5 \leq x \leq 1\end{cases}
$$

if $T_{\lambda}$ restricted to $\left[0, T_{\lambda}(0.5)\right]$ is rescaled to $h_{\lambda}: I \rightarrow I$, then $h_{\lambda}$ is given by

$$
h_{\lambda}(x)= \begin{cases}\lambda x, & 0 \leq x \leq \frac{1}{\lambda} \\ 2-\lambda x, & \frac{1}{\lambda} \leq x \leq 1\end{cases}
$$

and the rescaling of $h_{\lambda}$ restricted to $\left[h_{\lambda}(1), 1\right]$ to $f_{\lambda}: I \rightarrow I$ (or the core of the corresponding tent map $T_{\lambda}$ ) is given by

$$
f_{\lambda}(x)= \begin{cases}\lambda x+(2-\lambda), & 0 \leq x \leq \frac{\lambda-1}{\lambda} \\ -\lambda x+\lambda, & \frac{\lambda-1}{\lambda} \leq x \leq 1\end{cases}
$$

In the following, $c_{\lambda}$ will denote the critical point $\frac{\lambda-1}{\lambda}$ of $f_{\lambda}$. A parameter value $\lambda \in[1,2]$ is periodic if $c_{\lambda}$ is periodic under $f_{\lambda}$, and prefixed if $c_{\lambda}$ maps to a fixed point under some iterate of $f_{\lambda}$. The periodic parameters are dense in [1,2], and the prefixed parameters are dense in $[\sqrt{2}, 2]$ (see, for example, [7] or [5]).

Since $(I, f)=\left(J,\left.f\right|_{J}\right)$ where $J=\bigcap_{n \geq 0} f^{n}(I),\left(I, T_{\lambda}\right)$ is homeomorphic with $\left(I, h_{\lambda}\right)$, while $\left(I, h_{\lambda}\right)$ consists exactly of a homeomorphic copy of $\left(I, f_{\lambda}\right)$ and an infinite ray entwined with $\left(I, f_{\lambda}\right)$.

The first result indicates that the inverse limit space $\left(I, f_{\lambda}\right)$ is locally wellbehaved if the orbit of the critical point $c_{\lambda}$ is finite (thus for a dense set of parameter values).

Theorem 1. Let $\sqrt{2} \leq \lambda \leq 2$, and suppose that $\left\{f_{\lambda}^{n}\left(c_{\lambda}\right)\right\}_{n \in \mathbb{N}}$ is finite. Then all but finitely many points of $\left(I, f_{\lambda}\right)$ have a neighbourhood that is homeomorphic with the product of a Cantor set and an arc.

Proof. Let $I_{i}, 1 \leq i \leq k$, be the closures of the components of $I \backslash\left\{f_{\lambda}^{n}\left(c_{\lambda}\right)\right\}_{n \in \mathbb{N}}$. The $I_{i}$ 's form a Markov partition for $f_{\lambda}$. That is, for each $i,\left.f_{\lambda}\right|_{I_{i}}$ is one-to-one and $f_{\lambda}\left(I_{i}\right)$ is a union of a subcollection of $\left\{I_{j}\right\}_{j \leq k}$. 
For each $i \leq k$, let $f_{\lambda, i}^{-1}$ denote the inverse of $\left.f_{\lambda}\right|_{I_{i}}$. Suppose $\underline{x}=\left(x_{0}, x_{1}, \ldots\right) \in$ $\left(I, f_{\lambda}\right)$ is such that $x_{N} \notin\left\{f_{\lambda}^{n}\left(c_{\lambda}\right)\right\}_{n \in \mathbb{N}}$ for some $N \in \mathbb{N}$. Then $x_{N} \in \operatorname{int}\left(I_{j}\right)$ for some $j$. Let $\Sigma=\left\{\left(a_{1}, a_{2}, \ldots\right): a_{l} \leq k\right.$ for each $l \in \mathbb{N}, f_{\lambda}\left(I_{a_{1}}\right) \supseteq I_{j}, f_{\lambda}\left(I_{a_{l+1}}\right) \supseteq I_{a_{l}}$ for each $l\}$. Since $f$ is transitive for $\lambda \geq \sqrt{2}, \Sigma$, with the product topology, is a Cantor set. The set $U=\Pi_{N}^{-1}\left(\operatorname{int}\left(I_{j}\right)\right)$ is a neighbourhood of $\underline{x}$ and $\phi: \Sigma \times \operatorname{int}\left(I_{j}\right) \mapsto U$ defined by $\phi\left(\left(a_{1}, a_{2}, \ldots\right), t\right)=\left(f_{\lambda}^{N}(t), \ldots, f_{\lambda}(t), t, f_{\lambda, a_{1}}^{-1}(t), f_{\lambda, a_{2}}^{-1}\left(f_{\lambda, a_{1}}^{-1}(t)\right), \ldots\right)$ is a homeomorphism.

Most of the full attracting sets for maps in the Hénon family are homeomorphic to inverse limits of associated quadratic maps where the critical point has a finite orbit ([1]). According to [8], if the kneading sequence (defined following the next result) of the quadratic map appears as a kneading sequence for a map in the tent family, the inverse limit spaces for these two maps are homeomorphic, thus the full attracting set for the Hénon map has the structure described by Theorem 1. For a large number of the quadratic maps of interest, the kneading sequence does not appear in the tent family; in this case, the techniques of [1] can be used to prove that the full attracting sets also have this local structure.

The following lemma is a consequence of [3, Theorem 3].

Lemma 2. For $\left\{\lambda_{n}\right\}_{n=1}^{\infty} \subseteq[1,2]$, there is a sequence $\epsilon_{n}>0, n \in \mathbb{N}$, such that if $\left|\eta_{n}-\lambda_{n}\right|<\epsilon_{n}$, for $n \in \mathbb{N}$, then the inverse limit of

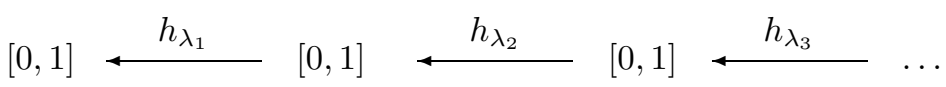

is homeomorphic with the inverse limit of

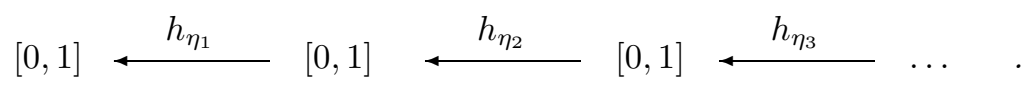

We need the language of unimodal maps and kneading theory for Lemma 3. A map $f: I \rightarrow I$ is unimodal if there is $c \in(0,1)$ such that $f$ is strictly increasing on $[0, c)$ and strictly decreasing on $(c, 1]$; the point $c$ is the critical point of $f$. We recall some basic notions of kneading theory for unimodal maps; for more details see [6]. For each $x \in[0,1]$, the itinerary of $x$ under the unimodal map $f$ is given by $I(x)=b_{0} b_{1} b_{2} \ldots$, where $b_{i}=R$ if $f^{i}(x)>c, b_{i}=L$ if $f^{i}(c)<c$, and $b_{i}=C$ if $f^{i}(x)=c$, with the usual convention that the itinerary stops after the first $C$. The kneading sequence of the map $f$, denoted $K(f)$, is defined to be the itinerary of $f(c)$. The parity-lexicographical ordering is put on the set of itineraries as follows. Set $L<C<R$. Let $W=w_{1} w_{2} \ldots$ and $V=v_{1} v_{2} \ldots$ be two distinct itineraries and let $k$ be the first index where the itineraries differ. If $k=1$, then $W<V$ iff $w_{1}<v_{1}$. If $k>1$ and $w_{1} \ldots w_{k-1}=v_{1} \ldots v_{k-1}$ has an even number of $R$ 's, i.e., has even parity, then $W<V$ iff $w_{k}<v_{k}$; if $w_{1} \ldots w_{k-1}$ has an odd number of $R$ 's, then $W<V$ iff $v_{k}<w_{k}$. It is an elementary fact that the map $x \mapsto I(x)$ is monotone, i.e., $x<y$ implies that $I(x) \leq I(y)$ [6, Lemma II.1.3]. As $f(c)$ is the maximum value of the function, it follows that if $A=a_{0} a_{1} \ldots=K(f)$, then any shift of $A\left(a_{j} a_{j+1} \ldots\right.$ for $\left.j \geq 0\right)$ is less than or equal to the kneading sequence itself in the parity-lexicographical order, that is, $A$ is shift maximal. If $\lambda \in[1,2]$ and $f_{\lambda}$ is as defined above, the map $\lambda \mapsto K\left(f_{\lambda}\right)$ is strictly increasing (see, for example, the appendix of [4]). 
Recall that $c_{\lambda}$ is the critical point of $f_{\lambda}$. Define $A_{a, \delta}=\{\lambda \in[\sqrt{2}, 2]$ : there exist $n$ and $0<a_{\lambda}<b_{\lambda}$ such that $f_{\lambda}^{n}(0) \in(a-\delta, a+\delta), f_{\lambda}^{n}\left(a_{\lambda}\right)=0, f_{\lambda}^{n}\left(b_{\lambda}\right)=c_{\lambda}$, and $f_{\lambda}^{n}$ is monotone on each of $\left.\left[0, a_{\lambda}\right],\left[a_{\lambda}, b_{\lambda}\right]\right\}$. In the proof of the next result about $A_{a, \delta}$, we make use of the notion of skeleton maps. For $n \geq 1$ and $\lambda \in[\sqrt{2}, 2]$, the $n^{\text {th }}$ skeleton map $\varphi_{n}$ is defined by $\varphi_{n}(\lambda)=T_{\lambda}^{n}(c)$, where $c=0.5$ is the critical point of $T_{\lambda}$. According to [7, §5], each $\varphi_{n}$ is continuous, piecewise polynomial, and differentiable except at periodic parameters with period less than $n$. Also, $\varphi_{n}^{\prime}$ is never zero, so $\varphi_{n}$ is strictly monotone on intervals containing no parameters with period less than $n$.

Lemma 3. For $a \in[0,1]$ and $\delta>0, A_{a, \delta}$ contains a dense open subset of $[\sqrt{2}, 2]$.

Proof. Since the periodic parameters are dense in [1,2], given a parameter value $\lambda$ and $\delta>0$, there are $n_{0}$ and $\lambda_{0}>\lambda$ such that $c_{\lambda_{0}}$ is periodic under $f_{\lambda_{0}}$ of period $k_{0} \geq n_{0}$ and if $f_{\gamma}$ has kneading sequence agreeing with that of $f_{\lambda_{0}}$ to $n_{0}-1$ places, then $|\lambda-\gamma|<\delta$. It follows from the fact that the prefixed parameters are dense in $[\sqrt{2}, 2]$ that $\lambda_{0}$ can be chosen so that $k\left(f_{\lambda_{0}}\right)=W C$ has even parity and the sequences $W R^{4} C$ and $W R^{3} C$ are shift maximal. Let $\gamma_{0}$ ( $\gamma_{1}$, respectively) denote the parameter value for which the associated tent (or core) map has kneading sequence $W R^{4} C$ (WR $R^{3} C$, respectively). Note that $\lambda_{0}<\gamma_{0}<\gamma_{1}<\lambda_{0}+\delta$ since $W C<W R^{4} C<W R^{3} C$. Also, there is no periodic kneading sequence between $W R^{4} C$ and $W R^{3} C$, which are of periods $k_{0}+4$ and $k_{0}+3$ respectively, with period less than $k_{0}+4$. That is, if $k=k_{0}+4$, then $T_{\gamma_{0}}^{k}(c)=c, T_{\gamma_{1}}^{k}(c)=T_{\gamma_{1}}\left(c_{\gamma_{1}}\right)$, and $\varphi_{n}$ is monotonically increasing between $\gamma_{0}$ and $\gamma_{1}$. In the language of the core map $f_{\gamma}$, $f_{\gamma_{0}}^{k}\left(c_{\gamma_{0}}\right)=c_{\gamma_{0}}, f_{\gamma_{1}}^{k}\left(c_{\gamma_{1}}\right)=1$, and $f_{\gamma}^{k}\left(c_{\gamma}\right)$ increases from $c_{\gamma_{0}}$ to 1 as $\gamma$ increases from $\gamma_{0}$ to $\gamma_{1}$.

For each $\gamma \in\left[\gamma_{0}, \gamma_{1}\right]$, the critical point $d_{\gamma}$ for $f_{\gamma}^{k}$ closest to and larger than $c_{\gamma}$ has itinerary $R W^{\prime} C$ where $W^{\prime}$ is the initial segment of $k\left(f_{\gamma_{0}}\right)$ of length $n_{0}+1$. That is, $f_{\gamma}^{k}\left(d_{\gamma}\right)=0, f_{\gamma}^{k}$ is monotone decreasing on $\left[c_{\gamma}, d_{\gamma}\right]$, and there is $e_{\gamma} \in$ $\left[c_{\gamma}, d_{\gamma}\right)$ such that $f_{\gamma}^{k}\left(e_{\gamma}\right)=c_{\gamma}$. Let $x_{\gamma}$ denote the nonzero fixed point for $f_{\gamma}$. Since $k\left(f_{\gamma}\right)=R L W^{\prime} \ldots>R L R^{\infty}, f_{\gamma}(0)$ with itinerary $W^{\prime} \ldots$ is less than $x_{\gamma}$ with itinerary $R^{\infty}$. Then there is $y_{\gamma} \in\left[e_{\gamma}, d_{\gamma}\right]$ such that $f_{\gamma}^{k+1}\left(y_{\gamma}\right)=x_{\gamma}$. It follows that as $\gamma$ increases from $\gamma_{0}, f_{\gamma}^{k+2}\left(c_{\gamma}\right)$ increases from 0 to $1, f_{\gamma}^{k+2}\left(e_{\gamma}\right)=0$, $f_{\gamma}^{k+2}\left(y_{\gamma}\right)=x_{\gamma}$, and $f_{\gamma}^{k+2}$ is monotone on each of $\left[c_{\gamma}, e_{\gamma}\right]$ and $\left[e_{\gamma}, y_{\gamma}\right]$. For some $\gamma^{\prime} \in\left[\gamma_{0}, \gamma_{1}\right], f_{\gamma}^{k+2}\left(c_{\gamma}\right) \in(a-\delta, a+\delta)$. Let $a_{\gamma}=f_{\lambda}^{2}\left(e_{\gamma}\right)$ and $z_{\gamma}=f_{\lambda}^{2}\left(y_{\gamma}\right)$. Then $f_{\gamma^{\prime}}^{k}(0) \in(a-\delta, a+\delta), f_{\gamma^{\prime}}^{k}\left(a_{\gamma}\right)=0, f_{\gamma^{\prime}}^{k}\left(z_{\gamma}\right)=x_{\gamma}$, and $f$ is monotone on each of $\left[0, a_{\lambda}\right],\left[a_{\lambda}, z_{\lambda}\right]$.

If $A=\bigcap_{a, \delta \in Q \cap[0,1]} A_{a, \delta}$, then $A$ contains a dense $G_{\delta}$. If $\lambda \in A$, then for any $a \in[0,1]$ and $\delta>0$, there are $n$ and $0<a_{\lambda}<b_{\lambda}$ such that $f_{\lambda}^{n}$ is monotone on each of $\left[0, a_{\lambda}\right],\left[a_{\lambda}, b_{\lambda}\right], f_{\lambda}^{n}(0) \in(a-\delta, a+\delta), f_{\lambda}^{n}\left(a_{\lambda}\right)=0$, and $f_{\lambda}^{n}\left(b_{\lambda}\right)=c_{\lambda}$. In particular, if $\eta \in A,\left\{f_{\eta}^{k}(c)\right\}_{k=1}^{\infty}$ is dense in $[0,1]$.

For $1<\eta \leq \sqrt{2}$, let $J_{0}=\left[f_{\eta}^{2}(1), 1\right]$ and $J_{1}=f_{\eta}\left(J_{0}\right)$. It is easily checked that the maps $\left.f_{\eta}^{2}\right|_{J_{i}}, i=0,1$ are conjugate (via affine homeomorphisms) with $f_{\eta^{2}}$. It follows that if $1<\eta \leq \sqrt{2}$ and $\eta^{2^{k}} \leq 2$, then $\left.f_{\eta}^{2^{k}}\right|_{J_{i}}$ is conjugate with $f_{\eta^{2^{k}}}$, where $J_{0}=\left[f_{\eta^{2^{k}}}(1), 1\right]$ and $J_{i}=f_{\eta}^{i}\left(J_{0}\right), 1 \leq i \leq 2^{k-1}$. Thus, for such $\eta,\left(I, f_{\eta}\right)$ contains $2^{k}$ homeomorphic copies of $\left(I, f_{\eta^{2^{k}}}\right)$. In light of this, the set $A$ in Theorems 4,5 , 
and 9 and Corollary 6 can be replaced by $A^{\prime}=\left\{\eta \in[1,2]: \eta^{2^{k}} \in A\right.$ for some $k \in \mathbb{N}\}$. Note that $A^{\prime}$ contains a dense $G_{\delta}$ subset of $[1,2]$.

Theorem 4. Suppose that $\eta \in A$, and let $\lambda \in(1,2]$. Given any subcontinuum $C$ of $\left(I, f_{\lambda}\right)$, there is a subcontinuum of $\left(I, f_{\eta}\right)$ homeomorphic with $C$.

Proof. Let $\epsilon_{n}>0, n \in \mathbb{N}$, be as in Lemma 2 with $\lambda_{n}=\lambda$ for all $n \in \mathbb{N}$. Let $b_{1}=c_{\lambda}$, and choose $a_{1} \in\left(0, b_{1}\right)$ such that $\left|1+\frac{a_{1}}{b_{1}}-\lambda\right|<\epsilon_{1}$. There is $\delta_{1}>0$ such that $a_{1}+\delta_{1}<b_{1}$ and if $\left|a-a_{1}\right|<\delta_{1}$, then $\left|1+\frac{a}{b_{1}}-\lambda\right|<\epsilon_{1}$. Let $n_{1}$ and $b_{2}$ be such that $\left|f_{\eta}^{n_{1}}(0)-a_{1}\right|<\delta_{1}, f_{\eta}^{n_{1}}\left(b_{2}\right)=b_{1}$ and $f_{\eta}^{n_{1}}$ has precisely one critical point in $\left(0, b_{2}\right)$ which maps to 0 under $f_{\eta}^{n_{1}}$. Now let $a_{2} \in\left(0, b_{2}\right)$ be such that $\left|1+\frac{a_{2}}{b_{2}}-\lambda\right|<\epsilon_{2}$, and choose $\delta_{2}$ small enough so that $a_{2}+\delta_{2}<b_{2}$ and if $\left|a-a_{2}\right|<\delta_{2}$, then $\left|1+\frac{a}{b_{2}}-\lambda\right|<\epsilon_{2}$. Continuing, $n_{2}$ and $b_{3}$ can be chosen such that $\left|f_{\eta}^{n_{2}}(0)-a_{2}\right|<\delta_{2}, f_{\eta}^{n_{2}}\left(b_{3}\right)=b_{2}$ and $f_{\eta}^{n_{2}}$ has precisely one critical point in $\left(0, b_{3}\right)$ which maps to 0 under $f_{\eta}^{n_{2}}$. We prove that if $b_{k}$ and $n_{k}$ are defined in this manner, then the inverse limit of

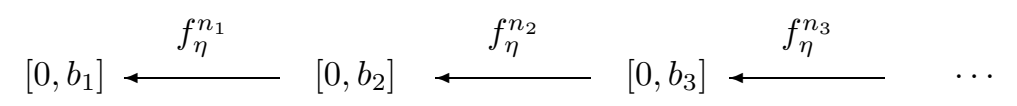

is homeomorphic with $\left(I, h_{\lambda}\right)$.

For $b>0$, define $\psi_{b}:[0, b] \rightarrow[0,1]$ by $\psi_{b}(x)=1-x / b$. For $k \in \mathbb{N}$, let $g_{k}=\psi_{b_{k}} \circ f_{\eta}^{n_{k}} \circ \psi_{b_{k+1}}^{-1}$. The commuting diagram
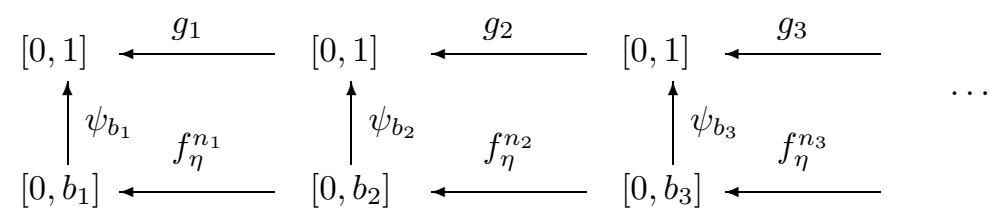

shows that the inverse limit of $\left\{g_{k}\right\}_{k=1}^{\infty}$ is homeomorphic to the inverse limit of $\left\{\left.f_{\eta}^{n_{k}}\right|_{\left[0, b_{k+1}\right]}\right\}_{k=1}^{\infty}$.

Claim. For each $k$ there is $\lambda_{k}$ such that $g_{k}=h_{\lambda_{k}}$ and $\left|\lambda_{k}-\lambda\right|<\epsilon_{k}$.

Proof of claim. It is easy to check that $g_{k}$ is a piecewise linear unimodal map with the critical point mapping to a maximum at 1 and the magnitude of the slopes of the linear pieces equal, say, to $\lambda_{k}$. Also, $g(0)=0$, so $g_{k}=h_{\lambda_{k}}$. Let $m=\eta^{n_{k}}$ (the magnitude of the slopes of the linear pieces of $\left.f_{\eta}^{n_{k}}\right)$ and $a=f_{\eta}^{n_{k}}(0)$. Then $m=\frac{a+b_{k}}{b_{k+1}}$, so $\lambda_{k}=b_{k+1}(m) \frac{1}{b_{k}}=\frac{a+b_{k}}{b_{k}}=1+\frac{a}{b_{k}}$. Since $\left|a-a_{k}\right|<\delta_{k},\left|\lambda_{k}-\lambda\right|=\left|1+\frac{a}{b_{k}}-\lambda\right|<\epsilon_{k}$. The claim is proved.

It follows that the inverse limit of $\left\{\left.f_{\eta}^{n_{k}}\right|_{\left[0, b_{k+1}\right]}\right\}_{k=1}^{\infty}$ is homeomorphic with the inverse limit of $\left\{h_{\lambda_{k}}\right\}_{k=1}^{\infty}$, which is homeomorphic with $\left(I, h_{\lambda}\right)$ by Lemma 2 .

Define $X$ to be the following subcontinuum of $\left(I, f_{\eta}\right)$ :

$$
\underline{x} \in X \leftrightarrow \Pi_{N_{i}}(\underline{x}) \in\left[0, b_{i+1}\right], i=0,1,2, \ldots,
$$

where $N_{0}=0, N_{i}=\sum_{k=1}^{i} n_{k}$ for $i \in \mathbb{N}$. Then $X$ is homeomorphic with the inverse limit of $\left\{\left.f_{\eta}^{n_{k}}\right|_{\left[0, b_{k+1}\right]}\right\}_{k=1}^{\infty}$, hence with $\left(I, h_{\lambda}\right)$. Since every subcontinuum of $\left(I, f_{\lambda}\right)$ is homeomorphic with a subcontinuum of $\left(I, h_{\lambda}\right)$, the theorem is proved.

In fact, the bonding map can be varied in Theorem 4 .

Theorem 5. Given $\eta \in A,\left\{\lambda_{n}\right\}_{n \in \mathbb{N}} \subseteq[1,2]$ and any subcontinuum $C$ of the inverse limit of $\left\{f_{\lambda_{n}}\right\}_{n=1}^{\infty}$, there is a subcontinuum of $\left(I, f_{\eta}\right)$ homeomorphic with $C$. 
Proof. First we show that given $\left\{\lambda_{n}\right\}_{n=1}^{\infty} \subseteq[1,2]$, there is $\left\{\eta_{n}\right\}_{n=1}^{\infty} \subseteq(1,2]$ such that the inverse limit of $\left\{f_{\lambda_{n}}\right\}_{n=1}^{\infty}$ is homeomorphic with a subcontinuum of the inverse limit of $\left\{h_{\eta_{n}}\right\}_{n=1}^{\infty}$. According to Lemma 2, we can assume without loss of generality that $\lambda_{n} \in(1,2]$ for each $n$. Given $a \in[0,1)$, define $g_{a}:[a, 1] \rightarrow[0,1]$ by $g_{a}(x)=\frac{1}{1-a}(x-a)$. Then $g_{a}^{-1}(x)=(1-a) x+a$.

Claim. Given $\lambda \in(1,2], a_{1} \in[0,1)$ and $\eta=2-a_{1}$, there is $a_{2} \in[0,1)$ such that

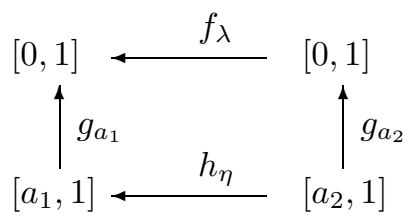

is a commutative diagram of surjections.

Proof of claim. Let $a_{2}=1-\frac{1-a_{1}}{2-a_{1}} \lambda$. Since $a_{1} \in[0,1), 0<\frac{1-a_{1}}{2-a_{1}} \leq 1 / 2$, so that $a_{2} \in[0,1)$. Thus $g_{a_{1}}^{-1} \circ f_{\lambda} \circ g_{a_{2}}$ is a piecewise linear unimodal map whose slope in absolute value is constant and equal to $\left(1-a_{1}\right)(\lambda)\left(\frac{1}{1-a_{2}}\right)=2-a_{1}=\eta$. Since $h_{\eta}(1)=a_{1}=g_{a_{1}}^{-1} \circ f_{\lambda} \circ g_{a_{2}}(1)$, the diagram must commute, with $h_{\eta}\left(\left[a_{2}, 1\right]\right)=$ $g_{a_{1}}^{-1} \circ f_{\lambda} \circ g_{a_{2}}\left(\left[a_{2}, 1\right]\right)=\left[a_{1}, 1\right]$. The claim is established.

Given the sequence $\left\{\lambda_{n}\right\}_{n=1}^{\infty}$, define $a_{1}=2-\eta_{1}$ and $\eta_{1}=\lambda_{1}$. Then $\eta_{1} \in(1,2]$ and $a_{1} \in[0,1)$. Assume that $a_{n} \in[0,1)$ and $\eta_{n}=2-a_{n} \in(1,2]$ have been defined. Let $a_{n+1}=1-\frac{1-a_{n}}{2-a_{n}} \lambda_{n}$ and $\eta_{n+1}=2-a_{n+1}$. Then, by the claim, $a_{n+1} \in[0,1)$ and $\eta_{n+1} \in(1,2]$. The commutative diagram

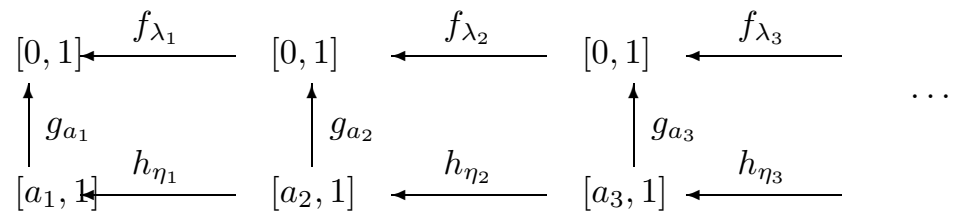

induces a homeomorphism between the inverse limit of $\left\{f_{\lambda_{n}}\right\}_{n=1}^{\infty}$ and the inverse limit of $\left\{\left.h_{\eta_{n}}\right|_{\left[a_{n+1}, 1\right]}\right\}_{n=1}^{\infty}$, a subcontinuum of the inverse limit of $\left\{h_{\eta_{n}}\right\}_{n=1}^{\infty}$.

Given the sequence $\eta_{n}$, the proof of Theorem 4 can be modified slightly to construct a subcontinuum of $\left(I, f_{\eta}\right)$ homeomorphic to the inverse limit of $\left\{h_{\eta_{n}}\right\}_{n=1}^{\infty}$. Specifically, given $\epsilon_{k}, \eta_{k}$ and $b_{k}$, there is $a_{k}$ such that $\left|1+\frac{a_{k}}{b_{k}}-\eta_{k}\right|<\epsilon_{k}$. Define $\delta_{k}, n_{k}$ and $b_{k+1}$ as before. Then maps $g_{k}=h_{\gamma_{k}}$ can be defined so that $\left|\gamma_{k}-\eta_{k}\right|<\epsilon_{k}$, hence the inverse limit of $\left\{h_{\gamma_{k}}\right\}_{k=1}^{\infty}$ is homeomorphic to the inverse limit of $\left\{h_{\eta_{k}}\right\}_{k=1}^{\infty}$, and the inverse limit of $\left\{g_{k}\right\}_{k=1}^{\infty}$ (that is, the inverse limit of $\left.\left\{h_{\gamma_{k}}\right\}_{k=1}^{\infty}\right)$ is homeomorphic to the inverse limit of $\left\{\left.f_{\eta}^{n_{k}}\right|_{\left[0, b_{k+1}\right]}\right\}_{k=1}^{\infty}$.

Corollary 6. Let $\eta \in A,\left\{\lambda_{n}\right\}_{n \in \mathbb{N}} \subseteq[1,2]$ and $U$ an open subset of $\left(I, f_{\eta}\right)$. For any subcontinuum $C$ of the inverse limit of $\left\{f_{\lambda_{n}}\right\}_{n=1}^{\infty}$, there is a subcontinuum $D$ of $\left(I, f_{\eta}\right)$ homeomorphic with $C$ with $D \subseteq U$.

Proof. Without loss of generality, $U$ is of the form $\left[U_{1} \times U_{2} \times \cdots \times U_{n} \times I \times \cdots\right] \cap$ $\left(I, f_{\eta}\right)$. It is enough to show that there is a subcontinuum $D^{\prime}$ of $\left(I, f_{\eta}\right)$ such that $D^{\prime}$ is homeomorphic to $\left(I, f_{\eta}\right)$ and $\Pi_{0}\left(D^{\prime}\right) \subseteq U_{n}$, since then $\Pi_{n}\left(\hat{f}_{\eta}^{n}\left(D^{\prime}\right)\right) \subseteq U_{n}$ and $\hat{f}_{\eta}^{n}\left(D^{\prime}\right) \subseteq U$ contains a subcontinuum homeomorphic with $C$. 
Since $\eta \in A,\left\{f_{\eta}^{k}\left(c_{\eta}\right)\right\}_{k=1}^{\infty}$ is dense in $[0,1]$. Choose $k \geq 2$ such that $f_{\eta}^{k}\left(c_{\eta}\right)=$ $f_{\eta}^{k-2}(0) \in U_{n}$. There is $v \in[0,1]$ such that $f_{\eta}^{k-2}[0, v] \subseteq U_{n}$. Let $b_{1}=v$ in the proof of Theorem 4 , and construct $\left\{b_{m}\right\}_{m=1}^{\infty} \subseteq[0,1],\left\{n_{m}\right\}_{m=1}^{\infty} \subseteq \mathbb{N}$ such that the inverse limit space $D^{\prime \prime}$ of $\left\{\left.f_{\eta}^{n_{k}}\right|_{\left[0, b_{m+1}\right]}\right\}_{m=1}^{\infty}$ is homeomorphic to $\left(I, f_{\eta}\right)$. Then $\Pi_{0}\left(\hat{f}_{\eta}^{k-2}\left(D^{\prime \prime}\right)\right)=f_{\eta}^{k-2}\left(\Pi_{0}\left(D^{\prime \prime}\right)\right) \subseteq f_{\eta}^{k-2}([0, v]) \subseteq U_{n}$.

Finally, we prove that if $\eta \in A$, then $\left(I, f_{\eta}\right)$ has uncountably many endpoints. A subcontinuum $T$ of a space $X$ is an end continuum in $X$ if whenever $T \subseteq A, T \subseteq B$ for continua $A, B \subseteq X$, then either $A \subseteq B$ or $B \subseteq A$. The point $x \in X$ is an endpoint of $X$ if $\{x\}$ is an end continuum in $X$.

Lemma 7. Suppose that $T$ is a subcontinuum of $(I, f)$ for a map $f: I \rightarrow I$, and that $0 \in \Pi_{n}(T)$ for infinitely many $n \in \mathbb{N}$. Then $T$ is an end continuum.

Proof. Let $A, B$ be subcontinua of $(I, f)$ with $T \subseteq A \cap B$. Then $0 \in \Pi_{n}(A)$ and $0 \in \Pi_{n}(B)$ for infinitely many $n$. It follows that either $\Pi_{n}(A) \subseteq \Pi_{n}(B)$ or $\Pi_{n}(B) \subseteq \Pi_{n}(A)$ infinitely often, hence for all $n$.

Lemma 8. Suppose that $(I, f) \supseteq T_{1} \supseteq T_{2} \supseteq \cdots$ where $T_{i}$ is an end continuum of $(I, f)$ for each $i$, and that $\bigcap_{i=1}^{\infty} T_{i}=\{\underline{x}\}$. Then $\underline{x}$ is an endpoint.

Proof. Let $A, B$ be subcontinua of $(I, f)$ with $\underline{x} \in A \cap B$. For each $i, T_{i} \cap A \cap B \neq \emptyset$. Since $(I, f)$ is atriodic, one of $T_{i}, A, B$ is contained in the union of the other two. If $T_{i} \subseteq A \cup B$ for any $i$, then, since $T_{i}$ is an end continuum, either $A \subseteq B$ or $B \subseteq A$, and the lemma is proved. If not, then without loss of generality, $A \subseteq T_{i} \cup B$ for infinitely many $i$, and $A \subseteq \bigcap_{i=1}^{\infty}\left(T_{i} \cup B\right)=B$.

If $\eta \in A$, then $c_{\eta}$ is recurrent, since $\left\{f_{\eta}^{k}\left(c_{\eta}\right)\right\}_{k=1}^{\infty}$ is dense in [0,1]. It follows from [2] that $\left(I, f_{\eta}\right)$ has an infinite number of endpoints. We can say more.

Theorem 9. For $\eta \in A,\left(I, f_{\eta}\right)$ has uncountably many endpoints.

Proof. Choose disjoint copies $T_{0}, T_{1}$ of $\left(I, f_{\eta}\right)$ contained in $\left(I, f_{\eta}\right)$. According to Corollary 6 , there are disjoint copies $T_{00}, T_{01}$ and $T_{10}, T_{11}$ of $\left(I, f_{\eta}\right)$ contained in $T_{0}$ and $T_{1}$ respectively so that $\operatorname{diam}\left(T_{s_{0} s_{1}}\right)<\frac{1}{2} \operatorname{diam}\left(T_{s_{0}}\right)$ for $s_{i} \in\{0,1\}$. Continuing in this manner, we can assign to each sequence $s_{0} s_{1} s_{2} \ldots$ of zeros and ones an infinite nested sequence of subcontinua $\left\{T_{s_{0} s_{1} \ldots s_{n}}\right\}_{n \in \mathbb{N}}$ of $\left(I, f_{\eta}\right)$ such that $T_{s_{0} s_{1} \ldots s_{n}}$ is homeomorphic to $\left(I, f_{\eta}\right)$ for each $n$ and $\operatorname{diam}\left(T_{s_{0} s_{1} \ldots s_{n}}\right) \rightarrow 0$ as $n \rightarrow \infty$. For each $n, T_{s_{0} s_{1} \ldots s_{n}}$ is not an arc, hence $0 \in \Pi_{k}\left(T_{s_{0} s_{1} \ldots s_{n}}\right)$ for infinitely many $k$, and $T_{s_{0} s_{1} \ldots s_{n}}$ is an end continuum. Since $\operatorname{diam}\left(T_{s_{0} s_{1} \ldots s_{n}}\right) \rightarrow 0$, for each sequence $s_{0} s_{1} s_{2} \ldots$, $\left|\bigcap_{n \in \mathbb{N}}\left\{T_{s_{0} s_{1} \ldots s_{n}}\right\}\right|=1$. Finally, if $s_{0} s_{1} s_{2} \ldots \neq t_{0} t_{1} t_{2} \ldots,\left(\bigcap_{n \in \mathbb{N}}\left\{T_{s_{0} s_{1} \ldots s_{n}}\right\}\right) \cap$ $\left(\bigcap_{n \in \mathbb{N}}\left\{T_{t_{0} t_{1} \ldots t_{n}}\right\}\right)=\emptyset$, thus there are an uncountable number of distinct endpoints.

We do not know whether the set of endpoints of $\left(I, f_{\lambda}\right)$ can be countably infinite.

\section{REFERENCES}

[1] M. Barge and S. Holte, Nearly one-dimensional Hénon attractors and inverse limits, Nonlinearity 8(1995), 29-42. MR 95m:58050

[2] M. Barge and J. Martin, Endpoints of inverse limit spaces and dynamics, Continua (eds. Cook et al.), Lecture Notes in Pure and Applied Math. 170(1995), Dekker, New York, 165182. MR 96b:54062

[3] M. Brown, Some applications of an approximation theorem for inverse limits, Proc. Amer. Math. Soc, 11(1960), 478-483. MR 22:5959 
[4] K.M. Brucks and B. Diamond, Monotonicity of auto-expansions, Physica D 51 (1991), 39-42. MR 92h:58101

[5] K.M. Brucks, B. Diamond, M.V. Otero-Espinar and C. Tresser, Dense orbits of critical points for the tent map, Contemporary Mathematics, 117 (1991), 57-61. MR 92e:58107

[6] P. Collet and J.P. Eckmann, Iterated Maps on the Interval as Dynamical Systems, Birkhauser, Boston, 1980. MR 82j:58078

[7] E.M. Coven, I. Kan and J.A. Yorke, Pseudo-orbit shadowing in the family of tent maps, Trans. Amer. Math. Soc. 308 (1988), 227-241. MR 90b:58236

[8] S. Holte, Inverse limits of Markov interval maps, preprint.

[9] C. Robinson, Dynamical Systems, CRC, Boca Raton, 1995.

[10] R.F. Williams, One-dimensional nonwandering sets, Topology 6 (1967), 473-487. MR 36:897

Department of Mathematics, Montana State University, Bozeman, Montana 59717

E-mail address: barge@math.montana.edu

Department of Mathematical Sciences, University of Wisconsin at Milwaukee, MilWAUKEE, WISCONSIN 53201

E-mail address: kmbrucks@alpha1.csd.uwm.edu

Department of Mathematics, University of Charleston, Charleston, South Carolina 29424

E-mail address: diamondb@ashley.cofc.edu 